Working Paper 97-66

Economics Series 32

September, 1997
Departamento de Economía

Universidad Carlos III de Madrid

Calle Madrid, 126

28903 Getafe (Spain)

Fax (341) 624-9875

\title{
MULTIPLE EQUILIBRIUM, VARIABILITY AND THE DEVELOPMENT PROCESS
}

\author{
Luis Carranza and José E. Galdón-Sánchez*
}

\begin{abstract}
Per capita output is more volatile in middle-income economies than in both low-income and high-income economies. In this paper, we address this fact in a two-periods overlapping generations model with two productive sectors (a developed sector and a subsistence sector) and a credit sector. In the second period, agents can choose to operate the developed technology. To do so, they can borrow resources to pay an entry cost. Due to the presence of an externality in the developed sector, as the fraction of managers in this sector increases, its productivity also increases. We show that, if the model economy is in the early or in the mature stages of development, there is a unique equilibrium. However, when the economy is in the middle stages of development, multiple equilibrium arise since the externality affects the performance of the credit market. The multiplicity of equilibria disappear when the credit market is perfect or it does not exist. Moreover, we find that in economies with imperfect credit markets, per capita output volatility tends to be higher than in economies with perfect or non-existent credit markets.
\end{abstract}

\section{Key Words}

Multiple equilibrium, externalities, market imperfections, sunspots, credit market, development.

*Carranza, International Monetary Fund (lcarranza @imf.org). Galdón-Sánchez, Universidad Carlos III de Madrid. We acknowledge very useful comments from Raouf Boucekkine, V.V. Chari, J.C. Conesa, J. Fdez-Villaverde, Nobu Kiyotaki, Ed Prescott, and seminar participants at the University of Minnesota Macro Workshop and the XV Latin American Meeting of the Econometric Society, Santiago, Chile. José E. Galdón-Sánchez thanks DGICYT for grant PB94-0378. Any views expressed herein are those of the authors and not necessarily those of the International Monetary Fund. The usual disclaimers apply. 


\section{Introduction}

The purpose of this paper is to address the high variability of per capita GDP displayed by middle-income countries, which contrasts with the low variability of capita GDP shown by both, low and high income economies. In order to illustrate this fact, Figure 1 plots the variability for different groups of countries ${ }^{1}$.

\section{[Figure 1 here]}

The paper also explores the relationship between the degree of development in the financial sector and per capita GDP variability. We found that, in economies where either a perfect credit market exists or the credit market is non-existent, the equilibrium is unique. However, when the credit market exists but is imperfect, there could be more than one equilibrium. In that case, the multiplicity of equilibria arises in middle stages of development.

In the presence of multiplicity of equilibria, we assume the existence of a sunspot process that coordinates the actions of the agents. In that respect, this paper is related to Cooper and Ejarque (1995), Sorger (1994), and Spear (1991). The paper by Cooper and Ejarque (1995) presents a model in which the indeterminacy of equilibrium is resolved by a sunspot process. There, the multiplicity of equilibria arises from the existence of non-convexities in the intermediation process. In Sorger (1994), a one-sector neoclassical growth model with borrowing constraints and heterogeneous agents is used to show that there can exist sunspot equilibria. Spear (1991) analyzes a dynamic model of pure capital accumulation to show the existence of sunspot equilibria in a way that avoids converting the model to an overlapping generations equivalent.

\footnotetext{
${ }^{1}$ The data used to draw Figure 1 come from the Summers and Heston Penn World Table 5.5 (1960-1990). OPEC members, countries with less than one million citizens and countries without a complete data set were dropped from the data. We first regressed per capita GDP against time for each country, calculating the GDP trend afterwards. The statistic we used consists of the sum of each country's squared per capita GDP deviations from its trend, divided by the average per capita GDP. These statistics yield variability indices for each one of the countries in our data set. Finally, countries are classified by income levels and an average variability index for each group of countries is obtained.
} 
In our model economy, there are two central elements: externalities and market imperfections. Both are necessary conditions for the existence of multiple equilibria. The way in which the sunspot mechanism affects the equilibrium will depend on both the degree of imperfection and the size of the economy.

In an economy with credit imperfections, the income distribution plays a very important role in determining the equilibrium. In that sense, this paper follows the line of work of Galor and Zeira (1993) and Banerje and Newman (1994), where income distribution is an important instrument to explain the economy's behavior in the development process. In our model, the income distribution and the externality effect determine the number of equilibria, and the stage at which the multiplicity of equilibria arises and when it vanishes.

In order to model this economy, we use an overlapping generations model. In each period, a continuum of agents of measure one is born and lives for two periods. In the first period, the agents are heterogeneous in their ability to work. In the second period, they can become entrepreneurs and must decide whether to use an advanced technology (for which they have to pay an entry cost) or a subsistence technology. They can borrow resources to pay the entry cost. In addition, there exists an externality in the advanced technology: the larger the fraction of people using the advanced technology, the more productive this technology becomes.

In our model, the financial market is incomplete. The reason is twofold. First, lenders can not force borrowers to repay their debts unless the debts are secured. Second, the returns from investment are only partially collateralizable. This implies that credit constraints arise endogenously. Given this credit market imperfection, the externality in the productive sector will be reflected in the financial sector: the larger the fraction of people using the advanced technology, the larger the fraction of people that will have access to the credit market. The reason for this is that the borrowing constraint is relaxed as the returns in the advanced technology increase. This is the source of multiple equilibrium in the model which arises in the middle stage of the development process. On the contrary, in early and mature stages of development, the multiplicity does not exist. 
It is worthwhile to notice that, if the credit market does not exist, there is no interaction between the externality in the productive sector and the borrowing constraint. Only the fraction of people with wealth greater than the cost of entry will become entrepreneurs. This is also true when the credit market is perfect. In the latter case, there are no borrowing constraints and all the resources are used to finance the payment of the entry cost for those who want to use the advanced technology. Moreover, the interest rate will be such that the agents are indifferent between using the advanced or the subsistence technology.

The paper shows that the introduction of a new "technology", a financial technology in our case, could affect positively the growth rate in the economy and, at the same time, be a source of higher variability if the markets are not complete. The Mexican crisis in a globalized market environment illustrates this point.

The paper is organized as follows. In Section 2, the environment is presented. In Section 3, the problem of occupational choice is discussed. In Section 4, we analyze the economy's labor and credit markets. Section 5 is devoted to the definition of equilibrium. Section 6 explains the relationship between the level and variability of GDP per capita in an economy with imperfect credit markets. In Section 7 , the behavior of wages, interest rates and entrepreneurial choice during the development process are analyzed. Section 8 discusses the cases of perfect credit market and non credit market. Finally, the conclusions are presented in Section 9.

\section{The Environment}

The model is a two-period overlapping generations model. At each date $t$, a continuum of agents of measure one is born. Each agent is endowed with one unit of labor at each period. In the first period, the individuals can only be workers. They have some ability $a$ with support on the interval $[\alpha, \beta]$, a continuous probability distribution function $h(a)$, and a continuous cumulative distribution function $H(a)$. In the second period, the agents can be managers (with the same ability) or they can remain as workers, but with the lowest level of ability, $\alpha$. At $t=0$, the initial 'old' is endowed with $a v_{0}$, where $a$ is the same random variable as before, and $v_{0}$ is a non-negative 
number.

The agents born at time $t$ receive utility only from consumption at $t+1$. This is an innocuous ${ }^{2}$ assumption that simplifies the algebra. Each agent can choose between managing an advanced or a subsistence technology. If the agent chooses to manage the subsistence technology, she will have access to the following production function:

$$
g(l)
$$

with $g_{l}>0$ and $g_{l l}<0$. Where $l$ stands for labor input, $g_{l}$ is the first derivative with respect to $l$, and $g_{l l}$ is the second derivative with respect to $l$.

If the agent wants to manage the advanced technology, she must pay an entry cost $\phi$ at the end of the initial period. That is, the agent must commit herself to this technology. In the second period, she will have access to a production function of the form:

$$
f(l, \lambda)
$$

with $f_{l}>0, f_{l l}<0, f_{\lambda}>0, f_{\lambda \lambda}<0$ and $f_{l \lambda}>0$; where $\lambda$ stands for the fraction of people who choose to be a manager in the advanced technology. There are two points worth noticing. First of all, the way in which the production function is specified implies the existence of an externality. The second point is that, the production function does not depend on capital in the advanced or the subsistence sectors. The results would be exactly the same if capital were included. What it is really important is that the production functions have decreasing returns to scale in labor ${ }^{3}$ since the managerial ability is also a productive factor that needs to be considered.

Two assumptions about the parameters of the model must be done:

Assumption 1.- Even if nobody is using the advanced technology, the net return of this technology is higher than the return of the subsistence technology. That is:

$$
f(l, 0)-\phi>g(l), \forall l
$$

\footnotetext{
2Innocuous for our multiple equlibrium result.

3 And capital if it were included.
} 
Assumption 2.- The entry cost belongs to the interval $(\alpha, \beta)$. More specifically:

$$
\alpha<\phi<\beta
$$

where $\alpha$ and $\beta$ are both positive numbers.

There also exists a storage technology. By using this technology, the agents can transform date $t$ goods into date $t+1$ goods at a rate of one-toone.

The individual can borrow resources to pay the entry cost, with the obligation to repay the loan next period. However, since financial markets are imperfect, there exists an enforcement problem. The lenders can not force the borrowers to repay the debt, but they can seize a fraction $\theta$ of the borrower's managerial income. Also, it is assumed that there exists perfect information about the initial wealth of each agent.

In this model, the decisions are sequential. Agents born in period $t$ work for managers who were born in period $t-1$. They are paid according to their ability. At the end of period $t$, they make their financial decisions (lend, borrow or invest in the storage technology) and decide whether or not to pay the entry cost. At period $t+1$, those who paid the entry cost can manage the advance technology, otherwise they manage the subsistence technology or remain as workers. Managers will hire labor at a competitive wage and, at the end of the period, will execute the financial obligations and consume whatever is left.

Notice that the decision to pay the entry cost must be taken at the end of the period, when the number of entrepreneurs is unknown. In order to solve this problem, we assume the existence of a sunspot process that coordinates the actions of the agents ${ }^{4}$. That is, the sunspot is generated from a correlated device from which the agents receive signals. The decisions taken by the agents are functions of the received signal (observed sunspot). In order to incorporate the sunspot to the model, let us define $\omega_{t}$ as the state of the

\footnotetext{
${ }^{4}$ We follow the same idea as Cooper and Ejarque (1995). Theirs, as ours, is inspired in the idea of Correlated Equilibrium (see Auman, 1987) as a Nash Equilibrium in which agent's actions are based on extrinsic noise.
} 
economy at time $t$; and $\Sigma_{t}$ as the set of possible sunspots at time $t$, where in fact this set is a function of the state of the economy, i.e. $\Sigma_{t}=\Sigma\left(\omega_{t}\right)$. Also, let $\sigma_{t}$ be an element of $\Sigma_{t}$. For completeness, let us define a probability distribution of $\Sigma_{t}$ denoted $\Psi_{t}$, i.e. $\Psi_{t} \in \Psi\left(\Sigma_{t}\right)$ where $\Psi(A)$ denotes the set of probability distributions over the set $A$.

\section{Optimal Behavior}

In this section, we are going to analyze the optimal decisions of an agent type $a$ born at period $t$. This is easy to do working the model backwards. Let us start with the assumption that the agent has paid the entry cost at time $t$. This means she is a manager in the advanced sector and she will try to maximize her managerial income given the wage rate, $v_{t+1}$. That is:

$$
\Pi_{a}\left(v_{t+1}, \lambda_{t+1}\right)=\max _{l_{a}}\left\{f\left(l_{a}, \lambda_{t+1}\right)-v_{t+1} l_{a}\right\}
$$

where $l_{a}\left(v_{t+1}, \lambda_{t+1}\right)$ is the solution to this problem. Notice that, at this stage, the fraction of total managers in the advanced sector is known.

If the agent did not pay the entry cost, she must decide between being a worker or a manager in the subsistence sector. The income from being a manager at the subsistence sector is:

$$
\Pi_{s}\left(v_{t+1}\right)=\max _{l_{s}}\left\{g\left(l_{s}\right)-v_{t+1} l_{s}\right\}
$$

where $l_{s}\left(v_{t+1}\right)$ is the solution to (6). Notice that in equilibrium $v$ will be a function of $\lambda$; so $l_{s}$ and $\Pi_{s}$ will also be functions of $\lambda$.

The income from being a worker is just:

$$
\Pi_{w}\left(v_{t+1}\right)=\alpha v_{t+1}
$$

From equations (6) and (7), we can find a threshold level for the wage, $v^{0}$, such that, for any $v<v^{0}$, the agents who did not pay the entry cost will prefer to be managers at the subsistence sector and, whenever $v>v^{0}$, those agents will prefer to be workers ${ }^{5}$. The existence and uniqueness of $v^{0}$ follow

\footnotetext{
${ }^{5}$ It is easy to see that when $\alpha \rightarrow 0, v^{0} \rightarrow \infty$. We will assume that this is the case.
} 
from the continuity and concavity of $g(l)$.

At the end of period $t$, an agent type $a$ has wealth $a v_{t}$. At that time, she must choose whether or not to pay the entry cost and the financial contracts. Notice that, at this point, $\lambda_{t+1}$ is unknown, but the agents observe a signal (sunspot) $\sigma$, and take a decision.

Given $\sigma$, the agents will pay the entry cost only if two conditions are satisfied:

$$
\begin{gathered}
\Pi_{a}(\lambda(\sigma))-r_{t, t+1} \phi \geq \max \left\{\Pi_{s}(\lambda(\sigma)), \Pi_{w}(\lambda(\sigma))\right\} \\
\theta \Pi_{a}(\lambda(\sigma)) \geq r_{t, t+1}\left(\phi-a v_{t}\right)
\end{gathered}
$$

where $r_{t, t+1}$ is the interest rate for loans made at period $t$ and repaid at period $t+1$. Interpreting equation (8) is trivial. It just tells us that the individual will pay the entry cost if the net return of doing this is higher than its opportunity cost. Equation (9) tells us about the borrowing constraint. If the amount of resources that the agent must repay at period $t+1$ is greater than the fraction $\theta$ of her final income, the agent will not receive credit because she will have an incentive to default.

Definition 1 . For an agent type $a$, and sunspot $\sigma$, let us define the payment function $\phi\left(a, \lambda(\sigma), v_{t}, v_{t+1}, r_{t, t+1}\right)$ such that:

$$
\begin{array}{r}
\phi\left(a, \lambda(\sigma), v_{t}, v_{t+1}, r_{t, t+1}\right)=1 \text { if }(8) \text { and (9) are satisfied } \\
=0 \text { otherwise }
\end{array}
$$

Let us now characterize the borrowing constraint of this economy. Using equation (9), we can have and idea of the extent of credit rationing in this economy. Solving equation (9) with equality, we get:

$$
a_{c}\left(\lambda(\sigma), v_{t}, r_{t, t+1}\right)=\frac{\phi-\frac{\theta \Pi_{a}(\lambda(\sigma))}{r_{t, t+1}}}{v_{t}}
$$

This means that, an agent with initial ability $a$, will receive credit if $a \geq$ $a_{c}\left(\lambda(\sigma), v_{t}, r_{t, t+1}\right)$ and she will be denied credit if $a<a_{c}\left(\lambda(\sigma), v_{t}, r_{t, t+1}\right)$. 
Now, we can see how the externality in the productive sector is reflected in the financial sector. The equation $a_{c}\left(\lambda(\sigma), v_{t}, r_{t, t+1}\right)$ is decreasing in $\lambda$, that is, the larger the fraction of population using the advanced technology, the more people will have access to the credit market. Also, note that $a_{c}\left(\lambda(\sigma), v_{t}, r_{t, t+1}\right)$ depends negatively on $v_{t}$. A higher wage implies a lower critical level, so the fraction of people who are credit constrained is smaller. In Proposition $1, a_{c}\left(\lambda(\sigma), v_{t}, r_{t, t+1}\right)$ will be completely characterized.

Proposition 1 . Let $a_{c}:[0,1] \times \Re \times[1, \infty) \rightarrow \Re$ be a function defined by (11). Then:

(i) $a_{c}(\cdot)$ is continuous in all its arguments,

(ii) $a_{c}(\cdot)$ is increasing in $r$, and

(iii) $a_{c}(\cdot)$ is decreasing in $\lambda$ and $v$.

Proof. [See Appendix]

\section{Credit, Investment and Labor}

Before defining the equilibrium for this economy, we will analyze in some detail the labor and the credit markets and also, how the problem of multiple equilibrium arises in this environment.

\subsection{Labor Market}

The labor market at period $t$ is comprised of managers (agents born at period $t-1$ ) who demand labor, and workers (agents born at $t$ and possibly at $t-1$ ) who supply labor. Since the size of each generation is one, the total labor supply of the new generation is given by:

$$
\int_{\alpha}^{\beta} a d H(a)=\delta
$$

Given the signal $\sigma$ at time $t$, some individuals will decide to become managers in the advanced sector paying the entry cost. Those who did not pay the fixed cost at time $t$ can either be workers or managers in the subsistence sector. The managers' labor demands in the advanced and the subsistence sectors are given by equations (13) and (14) respectively: 


$$
\begin{gathered}
\int_{\alpha}^{\beta} \phi\left(a, \lambda(\sigma), v_{t}, v_{t+1}, r_{t, t+1}\right) l_{a}(\lambda(\sigma)) d H(a)=\lambda(\sigma) l_{a}(\lambda(\sigma)) \\
\int_{\alpha}^{\beta}\left(1-\phi\left(a, \lambda(\sigma), v_{t}, v_{t+1}, r_{t, t+1}\right)\right) l_{s}(\lambda(\sigma)) d H(a)=(1-\lambda(\sigma)) l_{s}(\lambda(\sigma))
\end{gathered}
$$

The equilibrium wage in this market, $v^{*}$, is given (if $v^{*}<v^{0}$ ) by:

$$
\lambda(\sigma) l_{a}(\lambda(\sigma))+(1-\lambda(\sigma)) l_{s}(\lambda(\sigma))=\delta
$$

There could also be the case that the equilibrium wage, $v^{*}$, is greater than $v^{0}$. In that case, the agents who do not pay the entry cost become workers, and the equilibrium wage is given by:

$$
\lambda(\sigma) l_{a}(\lambda(\sigma))=\delta+(1-\lambda(\sigma)) \alpha
$$

As we pointed out in Section 3, the equilibrium wage in the labor market will be a function of the measure of entrepreneurs in the advanced sector. Assuming that $v(\lambda)<v^{0}$, we can use equations (5), (6) and (16) to solve for $v(\lambda), l_{a}(\lambda)$ and $l_{s}(\lambda)$. We found that $v(\lambda)$ and $l_{a}(\lambda)$ are strictly increasing in $\lambda$, and that $l_{s}(\lambda)$ is increasing in $\lambda$. A higher fraction of people using the advanced technology will imply an increment in the total labor demand, with the consequence of a higher level of wages. The next proposition formalizes this statement.

Proposition 2. If $v(\lambda)<v^{0} \forall \lambda$, then:

(i) $v(\lambda)$ is increasing in $\lambda$,

(ii) $l_{s}(\lambda)$ is decreasing in $\lambda$, and

(iii) $l_{a}(\lambda)$ is increasing in $\lambda$.

Proof. [See Appendix]

\subsection{Credit Market}

Since this is an overlapping generations model, there can not be debt contracts between different generations. All credit transactions must be realized 
among members of the same generation. So, at each period $t$, only the members of generation $t$ are engaged in financial transactions. Also, notice that the interest rate is bounded below by the storage technology gross return, i.e. $r_{t, t+1} \geq 1, \forall t$.

The total funds, at any point in time, are given by the total amount of resources in the economy, that is $\delta v_{t}$. Agents who want to be managers in the advanced technology sector at $t+1$ must pay $\phi$ at time $t$. They can do this using internal plus external funds. Therefore, the total demand for funds in this economy is given by:

$$
\phi \int_{\alpha}^{\beta} \phi\left(a, \lambda(\sigma), v_{t}, v_{t+1}, r_{t, t+1}\right) d H(a)=\phi \lambda(\sigma)
$$

Then, the equilibrium interest rate is given by:

$$
\phi \lambda_{t+1}\left(\sigma_{t}\right) \leq \delta v_{t} \text { with }=\text { if } r_{t, t+1} \geq 1
$$

In order to clarify equation (18), note that when we have borrowing constraints, the actual observed value of $\lambda_{t+1}$ is given by:

$$
\lambda_{t+1}=\int_{\alpha}^{\beta} \phi\left(a, \lambda_{t+1}, v_{t}, v_{t+1}, r_{t, t+1}\right) d H(a)=\int_{a_{c}\left(\lambda_{t+1}, v_{t}, r_{t, t+1}\right)}^{\beta} d H(a)
$$

Equation (19) is a fixed point problem with, possibly, more than one solution. Notice that, when $\phi \lambda_{t+1}<\delta v_{t}$, that is, when the fraction of entrepreneurs is less than the fraction of projects that can be financed in the economy, the interest rate will be equal to one. We can rule out some solutions of (19). In particular, we can rule out any $\lambda>\lambda_{\max }$, where $\lambda_{\max }$ is the maximum measure of people that can be managers at any time:

$$
\lambda_{\max , t}=\min \left(\frac{\delta v_{t}}{\phi}, 1\right)
$$

The total fraction of entrepreneurs is restricted by the resources in the economy, so it can not be greater than the funds available per project at any time, or the actual size of population. An equilibrium in which the resources are used in an efficient way, that is, to finance the maximum number of projects, can exist if and only if: 


$$
\lambda_{\max } \geq 1-H\left(a_{c}\left(\lambda_{\max }, v, 1\right)\right)
$$

If condition (21) is satisfied, there exists an excess of demand in the credit market. By continuity of $a_{c}(\cdot)$ and $H(\cdot)$, we can find an interest rate, $r\left(\lambda_{\max }, v\right)$, that will clear the market. The equilibrium will be reached if, at $r\left(\lambda_{\max }, v\right)$, the agents are indifferent between being a manager in the advanced and the subsistence technology; or because the tightening of the borrowing constraint will squeeze enough people out of the credit market, i.e.

$$
r\left(\lambda_{\max }, v\right)=\min \left(r_{1}\left(\lambda_{\max }, v\right), r_{2}\left(\lambda_{\max }, v\right)\right)
$$

where $r_{1}\left(\lambda_{\max }, v\right)$ and $r_{2}\left(\lambda_{\max }, v\right)$ solve the following equations:

$$
\begin{gathered}
\Pi_{a}\left(\lambda_{\max }\right)-\phi r_{1}\left(\lambda_{\max }, v\right)=\Pi_{s}\left(\lambda_{\max }\right) \\
\lambda_{\max }=1-H\left(a_{c}\left(\lambda_{\max }, v, r_{2}\left(\lambda_{\max }, v\right)\right)\right)
\end{gathered}
$$

Note that condition (21) can be satisfied only for some wage values. Before analyzing this situation, let us define the equilibrium properly.

\section{Equilibrium}

A sunspot-correlated equilibrium is sequences of sunspots $\left\{\sigma_{t}\right\}$, prices $\left\{r_{t, t+1}, v_{t}\right\}$, payment functions $\left\{\phi_{t}\left(a, \lambda_{t+1}\left(\sigma_{t}\right), v_{t}, v_{t+1}, r_{t, t+1}\right)\right\}$, fractions of managers $\left\{\lambda_{t}\left(\sigma_{t-1}\right)\right\}$, and labor demands $\left\{l_{a t}\left(\lambda_{t}\left(\sigma_{t-1}\right)\right), l_{s t}\left(\lambda_{t}\left(\sigma_{t-1}\right)\right)\right\}$ such that:

\section{Optimality}

- Given $\sigma_{t-1}, \lambda_{t}\left(\sigma_{t-1}\right), v_{t-1}, v_{t}$, and $r_{t-1, t} ; l_{a t}(\cdot)$ and $l_{s t}(\cdot)$ solve problems (5) and (6) respectively.

- Given $\sigma_{t-1}, \lambda_{t}\left(\sigma_{t-1}\right), v_{t-1}, v_{t}$, and $r_{t-1, t}$; an individual type $a$ chooses $\phi(\cdot)$ to satisfy $(8)$ and $(9)$.

\section{Market Clearing}


- Given $\sigma_{t}, \lambda_{t+1}\left(\sigma_{t}\right)$ and $v_{t} ; r_{t, t+1}$ and $v_{t+1}$ clear the credit and the labor markets.

\section{Consistency}

- Given $\sigma_{t}, v_{t}, v_{t+1}$ and $r_{t, t+1}$ :

$$
\int_{\alpha}^{\beta} \phi_{t}\left(a, \lambda_{t+1}\left(\sigma_{t}\right), v_{t}, v_{t+1}, r_{t, t+1}\right) d H(a)=\lambda_{t+1}\left(\sigma_{t}\right)
$$

\section{Multiple Equilibrium in the Development Process}

In this section, we study the process of economic development. We found that, in the early stages of development, there is only one equilibrium. The multiplicity of equilibria arises when the economy reaches a minimum level of wealth that allows a non-trivial fraction of people to obtain credit. Once the economy is at this stage, because of the existence of an externality in the productive sector, there will be more than one equilibrium. As we will prove in the next section, this problem does not arise if the credit market is perfect or if it does not exist. The reason for such result is that, in those cases, the connection between the externality effect and the credit market is broken. The multiplicity of equilibria will remain until the economy reaches a new threshold level. At that point, some individuals will have access to the credit market even though the measure of entrepreneurs is zero. by:

Before analyzing the development process, let us define the function $\gamma(\cdot)$

$$
\gamma(\lambda, v, r)=1-H\left(a_{c}(\lambda, v, r)\right)
$$

The next proposition characterizes this function $\gamma(\cdot)$. Note that the slope of this function with respect to $\lambda$ is always positive, but the magnitude of the slope will depend on the sign of the first derivative of $h(a)$ with respect to $a, h_{a}(a)$, and the relative strength of the externality effect.

Proposition 3 . The function $\gamma(\cdot)$ defined in (25) is: 
(i) A continuous function of $\lambda, v$ and $r$,

(ii) $\gamma(\cdot)$ is increasing in $\lambda$ and $v$,

(iii) $\gamma(\cdot)$ is decreasing in $r$, and

(iv) $\gamma_{\lambda}>0$, and $\gamma_{\lambda \lambda}>/<0$.

Proof. [See Appendix]

In what follows, we will do our analysis under the following assumptions:

Assumption 3.- $h(a)$ is uniform: $h_{a}(a)=0$ for all $a$.

Assumption 4.- There is a strong externality effect: $f_{\lambda}>B$, for some $B$ and $\lambda$ close to zero.

This is just for simplicity, the same results can be obtained if the income distribution is characterized by a negative slope, i.e. $h_{a}(a)<0$ for all $a$. In that case, along with Assumption 4, we have that $\gamma_{\lambda \lambda}<0$. If we choose a more "realistic" income distribution, with $h_{a}(a)>0$ for $a<x_{0}$ and $h_{a}(a)<0$ for $a>x_{0}$ for some $x_{0} \in(\alpha, \beta)$; the number of equilibria could increase and the multiplicity problem could be more persistent. The reason for this is that $\gamma_{\lambda \lambda}$ will be negative for $\lambda$ close to 0 , and positive for $\lambda$ close to 1 . The only different case would be when $x_{0}$ goes to $\beta$, i.e. $\gamma_{\lambda \lambda}>0$ for all $\lambda$. In that case, it can be proved that the multiplicity of equilibria will arise much later. In fact, if the externality effect is not too strong, the indeterminacy would appear, when in the case of the other economies, this problem has already disappeared.

Next, we will analyze the equilibrium along the development process for an economy that satisfies Assumptions 3 and 4. To find an equilibrium, we need to find the fixed point of equation (19). We can find a set of wages close to zero such that, even though the fraction of entrepreneurs is equal to one, there is no agent who can have access to the credit market. No matter how big the externality is, we can always have wages close enough to zero such that the critical level of ability to get credit is greater than $\beta$. In this situation, there exists a unique equilibrium in this economy. This equilibrium is given by $\lambda=0$.

For a higher wage, and given a strong externality effect, we can have a situation in which a positive fraction of entrepreneurs can overcome the 
credit constraint just because the same fraction of people becomes managers in the advanced technology sector. This will be an equilibrium if an only if the resources of the economy are big enough to finance this measure of entrepreneurs. If this is not the case, the economy has still a unique equilibrium. As the economy grows, we found two positive measures of entrepreneurs that could be considered as equilibria. We will show that, at some point, the resources in the economy will be big enough to finance the lowest of these fractions of managers. At that point, we have two equilibria. One given by the lowest of these positive measures. The other given by $\lambda=0$. Remember that even if the measure of entrepreneurs is zero, nobody can overcome the borrowing constraint.

From this period, the number of equilibria will increase to three being the first two as the ones obtained before, and the third one given by the resources in the economy: $\lambda_{t}=\frac{\delta v_{t}}{\phi}$. In this situation, such measure of entrepreneurs implies that a much bigger fraction of people can have access to the credit market. Since the resources in the economy are not big enough to finance all these projects, the interest rate must rise to clear this excess demand. We will prove that, for all the relevant range of wages, the highest fixed point of this problem will be always above the economy's resources. This situation will persist until the economy reaches some level of wage in which, even though nobody is using the advanced technology, the richest agent in the economy will be able to get credit. From this moment, the bad equilibrium $(\lambda=0)$ disappears. The unique equilibrium will be given by $\lambda=\lambda_{\max }$. Theorem 4 formalizes this statement.

Theorem 4 . If Assumptions 3 and 4 are satisfied, there exists two wage levels, $v_{1}$ and $v_{2}$, with $v_{1}>v_{2}$, such that:

$$
a_{c}\left(1, v_{1}, 1\right)=a_{c}\left(0, v_{2}, 1\right)=\beta
$$

Then:

(i) For any $v<v_{1}$, there exists only one equilibrium.

(ii) For some $v \in\left(v_{1}, v_{2}\right)$, there are multiple equilibria.

(iii) For any $v>v_{2}$, there exists only one equilibrium.

Proof. [See Appendix] 
In Figure 2, we show the evolution of the equilibria in the economy. For a given $v$, we can denote by $\lambda_{m}(v)$ and $\lambda_{M}(v)$ the lowest and the highest fixed points of equation (19), and by $R(v)$ the total resources per project in the economy. We see that $R(v)$ is always lower than $\lambda_{M}(v)$, which implies that $\lambda_{M}(v)$ will never be an equilibrium and that $\lambda_{m}(v)$ is decreasing. See Appendix for a formal proof of those statements.

[Figure 2 here]

Note that if we had a small open economy with perfect capital mobility, the highest fixed point, $\lambda_{M}(v)$, would be attainable. The implication of this is that an open economy will be expected to grow faster than a closed economy. Moreover, we should observe a higher variability for open economies than for closed ones.

Obviously, the variability in the economy during the multiple equilibrium stage will be higher than the variability in other stages. The degree of variability will depend on the sunspot probabilistic distribution. Since the equilibrium value of $\lambda$ is perfectly correlated with the sunspot, which is a random variable, then $\lambda$ is a random variable itself. In that sense, in this economy we can use the variance of $\lambda$ as a proxy for variability. The behavior of the variance of $\lambda$ is such that, when the multiplicity appears, the variance jumps from zero to a positive value. Then, the variance will be, first, decreasing with respect to $v$, starting to rise as the size of the economy increases. When the multiplicity disappears, the variance will be zero again. The reason for this behavior is that if two equilibria are close and one of them starts moving toward a third one, the variability decreases until they become too close. This is formalized in the next proposition.

Proposition 5. In the multiple equilibrium stage, the variability of the output, first decreases, and then increases.

Proof. [See Appendix]

In order to prove Proposition (5), we use a time-invariant probability distribution in which each sunspot has the same probability each period. The results are the same if we use symmetric first-order Markov probabilities in 
which the probability of observing a given sunspot this period will depend on the sunspot observed last period ${ }^{6}$. The results are not robust to all nonsymmetric Markov probabilities or other time-depending probabilities.

\section{Wages, Interest Rate and Entrepreneurship}

In this section, we will study the equilibrium values of wages, interest rates, and measure of entrepreneurs during the development process. We will prove that, if an economy satisfies some conditions for growth, then, for any initial condition $v_{0}$, the economy will converge to the long run equilibrium with positive probability. The condition for growth implies that there exists a required minimum level of productivity in the subsistence technology, and that the set of sunspots that implies the "good" equilibrium with probability zero has measure zero.

The conditions for growth are the following:

$$
g_{l}(\delta)>w^{\prime} \text { and } m(A)=0
$$

where $m(A)$ is the measure of a set $A$, and $A=\left\{\sigma_{t}: \operatorname{Pr}\left[\lambda\left(\sigma_{t}\right)=R\left(v_{t}\right)\right]=0\right\}$. The first part guarantees that the economy will reach the multiple equilibrium stage. The second implies that the economy will leave that stage.

If the conditions for growth are satisfied, the economy will reach the multiple equilibrium stage and, with positive probability, will be at the highest equilibrium. We can construct a sequence of $\lambda_{t}$ from $t$ equal one to $t$ equal $T$, in which $\lambda_{t}$ takes the highest value. This sequence has a positive probability if $T<\infty$. Since $\lambda_{t}$ is increasing at some finite time, the economy will leave this stage and will converge to the long run equilibrium: $\lambda^{*}=1, r^{*}=1$ and $v^{*}=f_{l}\left(\delta, \lambda^{*}\right)$. This fact is proved in the next theorem.

Theorem 6 . If the condition for growth is satisfied, for any initial $v_{0}$, the economy will converge to the long run equilibrium with positive probability. Proof. [See Appendix]

\footnotetext{
${ }^{6}$ By symmetric we mean that, the probability of today's sunspot $j$ given yesterday's sunspot $i$ is equal to the probability of today's sunspot $i$ given yesterday's sunspot $j$.
} 


\section{Credit Market and Variability}

The pourpouse of this section is to analyze how many different degrees of imperfection in the credit market will affect the equilibrium of the economy. In Section 6, we have already seen that, when there exists imperfections in the credit market (i.e. $0<\theta<1$ ), we could have multiple equilibria. Now, we show that when the credit market is perfect, i.e. $\theta=1$, or when the credit market does not exist, i.e. $\theta=0$, the equilibrium will be unique. The reason for this is that, when there is no credit market, there is no interaction between the externality in the productive sector and the borrowing constraint. Only the fraction of people with wealth greater than the entry cost will become entrepreneurs. Also, when the credit market is perfect, there are no longer borrowing constraints and, therefore, all the resources are used to finance the payment of the entry cost of those who want to use the advanced technology. The interest rate will be such that the agents are indifferent between using the advanced or the subsistence technology. Sections 8.1 and 8.2 will study both cases.

\subsection{Perfect-Credit Market Economy}

Suppose that $v_{0}<\frac{\delta}{\phi}$. This condition means that the initial resources are not enough to finance all the people in this economy. The people who become managers in the advanced technology sector are given by $\lambda_{0}=\frac{\delta v_{0}}{\phi}$. Since, by assumption, it is profitable to be a manager when the interest rate is one, everybody will demand credit for investment. In order for the credit market to clear, the interest rate will have to rise.

Once we have $\lambda_{0}$, the wage rate for the next period, $v_{1}$, can be uniquely determined using $v_{1}=v(\lambda)$ (see Section 4). Notice that, even though we have an externality in the productive sector, each time the measure of entrepreneurs is only determined by the resource constraints in the economy. The next proposition formalizes this statement.

Proposition 7 . Given some endowment av for the initial old such that $v_{0}<\frac{\phi}{\delta}$, if the credit market is perfect, then:

(i) At each $t$, there is only one equilibrium, i.e. $v_{t}, \lambda_{t+1}$, and $r_{t, t+1}$ are uniquely determined. 
(ii) The equilibrium sequences $\left\{v_{t}\right\}$ and $\left\{\lambda_{t}\right\}$ are non decreasing and converge to the long run equilibrium.

Proof. [See Appendix]

It is worthwhile to notice the behavior of the interest rate. When $\lambda<1$, $r_{t, t+1}(\lambda)$ is increasing in $\lambda$. The reason for this is that, as the total fraction of managers increases in this economy, the managerial profits also increase (due to the externality). As a result, the interest rate needed to clear this market up is higher. However, as soon as $\lambda$ reaches the value of one, the financial resources are abundant relative to the population resources (the fraction of managers can not be greater than one) and the interest rate will drop abruptly to one (the storage technology return). Notice that we can observe the same situation when there exists multiple equilibrium. In that case, the drastic change in interest rate will be accompanied by a drastic change in $\lambda$. However, when the credit market is perfect, a drastic change in interest rate will be caused by a change of regime. The economy moves from a resources constrained to a resources unconstrained economy. In that case, a large movement in the interest rate is accompanied by a small change in $\lambda$.

\subsection{Non Credit Market Economy}

Suppose now that the initial condition over $v_{0}$ is such that $\beta v_{0}<\phi$, which implies that nobody will become entrepreneur at period one. The wage rate at that period will be given by $v_{1}=g_{l}(\delta)$. In this case, the condition for growth is that $v_{1}>\frac{\phi}{\beta}$, otherwise the advanced technology will never be used. Noticed that, when there is no credit market or when the credit market is incomplete, there are some conditions on the productivity of the subsistence sector to guarantee that the economy will take off. This is not the case when the credit market is perfect.

Since the people can not borrow resources, the entry cost must be selffinanced. Then, given $v_{t}$, only a fraction of people equal to $1-H\left(\frac{\phi}{v_{t}}\right)$ is going to become managers the next period. Once $\lambda_{t-1}$ is known, the wage rate $v_{t+1}$ can be easily determined (see Section 4 ). Then, the sequences of wages $\left\{v_{t}\right\}$ and measures of managers $\left\{\lambda_{t}\right\}$ are uniquely determined, and they will converge to the long-run equilibrium. The next proposition formalizes 
this statement.

Proposition 8 . Given some endowment a $v_{0}$ for the initial old such that $v_{0}<\frac{\phi}{\beta}$, if the credit market does not exist and $g_{l}(\delta)>\frac{\phi}{\beta}$, then:

(i) At each period $t$, there is only one equilibrium, i.e. $v_{t}$ and $\lambda_{t+1}$ are uniquely determined.

(ii) The equilibrium sequences $\left\{v_{t}\right\}$ and $\left\{\lambda_{t}\right\}$ are non decreasing and converge to the long run equilibrium.

Proof. [See Appendix]

\section{Conclusion}

This paper explains the GDP variability pattern of an economy during the development process. It is found that, at the middle stages of development, the economy experiences high levels of GDP variability. On the other hand, in early and mature stages of development, we observe a much lower variability in per capita GDP. This variability is explained because, in an imperfect credit market environment, the number of equilibria will depend on the size of the economy. In particular, when the economy is very poor, there is only one possible equilibrium. After the economy reaches some threshold level, there can be multiple equilibria. This multiplicity disappears when the economy is fully developed. The existence of multiple equilibria is due to the fact that the externality in the productive sector will be reflected in the financial one. A larger fraction of people using the advanced technology will imply a larger fraction of people with access to the credit market.

The relationship between the degree of development of the financial sector and the variability in the economy is also analyzed. It is found that, in the case of either economies with perfect credit markets or economies in which the credit markets are non-existent, the equilibrium is unique. However, when the credit market exists but is imperfect, there could be more than one equilibrium. In the latter case, the multiplicity arises in the middle stages of development. This is due to the fact that when there is no credit market, there is no interaction between the externality in the productive sector and the borrowing constraint. Only the fraction of people with wealth 
greater than the entry cost will become entrepreneurs. Also, when the credit market is perfect, there are no borrowing constraints, and all the resources are used to finance the payment of the entry cost for those who want to use the advanced technology.

\section{References}

Aumann, R. (1987), Correlated Equilibrium as an Expression of Bayesian Rationality, Econometrica, vol. 55, pp. 1-18.

Banerje, A.V. and A.F. Newman (1993), Occupational Choice and the Process of Development, Journal of Political Economy, vol. 101, pp. 274298.

Carranza, L. (1996), Development Process with Imperfect Credit Market, Ph.D. Dissertation, University of Minnesota.

Carranza, L. and J.E. Galdón-Sánchez (1997), Institutional Change, Credit Imperfections and Development, manuscript, Universidad Carlos III de Madrid.

Cooper, R. and J. Ejarque (1995), Financial Intermediation and the Great Depression: A Multiple Equilibrium Interpretation, Carnegie-Rochester Conference Series on Public Policy, vol. 43, pp. 285-324.

De Long, J. and L. Summers(1984), The Changing Cyclical Variability of Economic Activity in the United States, NBER Working Paper No. 1450.

Galor, O. and J. Zeira (1993), Income Distribution and Macroeconomics, Review of Economic Studies, vol. 60, pp. 35-52.

Lucas, R. (1988), On the Mechanics of Economic Development, Journal of Monetary Economics, vol. 22, pp. 3-42.

Parente, S. and E. Prescott (1994), Barriers to Technology Adoption and Development, Journal of Political Economy, vol. 102, pp. 298-321.

Romer, C. (1986), Is the Stabilization of the Postwar Economy a Figment 
of the Data?, American Economic Review, vol. 76, pp. 314-334.

Sorger, G. (1994), On the Structure of Ramsey Equilibrium: Cycles, Indeterminacy, and Sunspots, Economic Theory, vol. 4, pp. 745-764.

Spear, S. (1991), Growth, Externalities, and Sunspots, Journal of Economic Theory, vol. 54, pp. 215-223. 


\section{APPENDIX}

Proof of Proposition 1: First of all, we need to prove that $\Pi_{a}(\lambda)$ is a continuous function of $\lambda$.

Claim: $\Pi_{a}(\lambda)$ is a continuous and increasing function of $\lambda$.

Proof. In order to prove this claim, we need to use a result that will be proved later (Proposition 2): $v(\lambda)$ is a continuous and increasing function of $\lambda$. Given that $f(l, \lambda)-v(\lambda) l$ is bounded from above, continuous in $l$ and $\lambda$, and with compact range, we can apply the Theorem of the Maximum to show that $\Pi_{a}(\lambda)$ is a continuous function. To see that it is increasing in $\lambda$, just take derivatives with respect to $\lambda$ and applying the Envelop Theorem we get:

$$
\frac{\partial \Pi(\lambda)}{\partial \lambda}=f_{\lambda}-\frac{\partial v(\lambda)}{\partial \lambda} l(v(\lambda), \lambda)
$$

This is positive if the average labor productivity is greater than the marginal labor productivity, which is the present case (see Proposition 2).

(i) By our claim, it is easy to see that $a_{c}(\cdot)$ is continuous in $\lambda, r$, and $v$.

(ii) Moreover, we can take partial derivatives with respect to $r$, and we get:

$$
\frac{\partial a_{c}(\cdot)}{\partial r_{t, t+1}}=\frac{\theta \Pi_{a}(\lambda)}{v_{t}\left(r_{t, t+1}\right)^{2}}>0
$$

(iii) Now, taking derivatives with respect to $\lambda$ and $v$, respectively:

$$
\begin{gathered}
\frac{\partial a_{c}(\cdot)}{\partial v_{t}}=\frac{-a_{c}(\cdot)}{v_{t} r_{t, t+1}}<0 \\
\frac{\partial a_{c}(\cdot)}{\partial \lambda}=\frac{-\theta}{v_{t} r_{t, t+1}}\left(\frac{\partial \Pi_{a}(\lambda)}{\partial \lambda}\right)<0
\end{gathered}
$$

Proof of Proposition 2: Given that all the conditions are satisfied for the Theorem of the Maximum, we can apply it to equations (5) and (6). After maximizing, we get the following equations: 


$$
\begin{gathered}
f_{l}\left(l_{a}(v, \lambda), \lambda\right)=v \\
g_{l}\left(l_{s}(v)\right)=v
\end{gathered}
$$

Now, using the equilibrium market condition (15), we have a system of three equations with three unknowns: $l_{a}(\lambda, \delta), l_{s}(\lambda, \delta)$ and $v(\lambda, \delta)$. This can be reduced to a system of two equations (solving for $l_{s}(\lambda, \delta)$ ). Taking derivatives with respect to $\lambda$, we get:

$$
\left(\begin{array}{cc}
0 & f_{l l} \\
1+\frac{g l \lambda}{f_{l l}(1-\lambda)} & \frac{g_{l l \lambda}}{(1-\lambda)}
\end{array}\right)\left(\begin{array}{c}
\frac{\partial v}{\partial \lambda} \\
\frac{\partial l_{a}}{\partial \lambda}
\end{array}\right)=\left(\begin{array}{c}
-f_{l \lambda} \\
0
\end{array}\right)
$$

Solving (36), we have:

$$
\left(\begin{array}{l}
\frac{\partial v}{\partial \lambda} \\
\frac{\partial \ell_{a}}{\partial \lambda}
\end{array}\right)=\frac{1}{|A|}\left(\begin{array}{c}
\frac{-g_{l l} f_{l \lambda} \lambda}{(1-\lambda)} \\
f_{l \lambda}\left(1+\frac{g_{l 1 \lambda}}{f_{l l}(1-\lambda)}\right)
\end{array}\right)>\left(\begin{array}{l}
0 \\
0
\end{array}\right)
$$

Where $|A|=-\left(f_{l l}+\frac{g_{l l \lambda}}{(1-\lambda)}\right)>0$. This proves (i) and (iii). In order to prove (ii), just note that from equation (15):

$$
\frac{\partial l_{s}(\cdot)}{\partial \lambda}=\frac{-(1-\lambda)}{\lambda} \frac{\partial l_{a}(\cdot)}{\partial \lambda}
$$

\section{Proof of Proposition 3:}

(i) The continuity of $\gamma(\cdot)$ follows from the continuity of $H(\cdot)$ and the continuity of $a_{c}(\cdot)$.

(ii) Since $a_{c}(\cdot)$ is decreasing in $\lambda$ and $v$, and $H(\cdot)$ is increasing in $a$, this proves that $\gamma(\cdot)$ is increasing in $\lambda$ and $v$.

(iii) To prove that $\gamma(\cdot)$ is decreasing in $r$, just note that $a_{c}(\cdot)$ is increasing in $r$ and $H(\cdot)$ is increasing in $a$.

(iv) Taking derivatives with respect to $\gamma(\cdot)$ :

$$
\frac{\partial \gamma(\cdot)}{\partial \lambda}=-h\left(a_{c}(\lambda, v, r)\right) \frac{\partial a_{c}(\lambda, v, r)}{\partial \lambda}>0
$$

Equation (39) is positive because of Proposition 1 part (iii). 


$$
\frac{\partial^{2} \gamma(\cdot)}{\partial \lambda^{2}}=-h_{a}\left(a_{\mathrm{c}}(\lambda, v, r)\right) \frac{\partial a_{c}(\lambda, v, r)}{\partial \lambda}-h\left(a_{\mathrm{c}}(\lambda, v, r)\right)\left(\frac{\partial a_{c}(\lambda, v, r)}{\partial \lambda}\right)^{2}
$$

The second term is always negative and the first term could be positive (if $h_{a}(a)>0$ ), negative (if $h_{a}(a)<0$ ), or zero (if $h_{a}(a)=0$ ). The overall result will be unknown. If the externality effect is almost null, and $h_{a}(a)>0$, then (40) can be positive. But if the externality effect is strong, the second term will dominate the first one and (40) will be negative.

\section{Proof of Theorem 4:}

(i) For any $v<v_{1}$, we have that $a_{c}(1, v, 1)>\beta$. Then $\gamma(\lambda, v, 1)=0$ for any $\lambda$. The only equilibrium is $\lambda=0$.

(ii) Because of (26) and the continuity of $a_{c}(\cdot)$, we can define a function $\lambda^{z}(v)$ for $v \in\left(v_{1}, v_{2}\right)$ such that:

$$
a_{c}\left(\lambda^{z}(v), v, 1\right)=\beta
$$

Notice that $\lambda^{z}(\cdot)$ is decreasing in $v, \gamma_{\lambda}(\cdot)=0$ for $\lambda \leq \lambda^{z}(v)$ and $\gamma_{\lambda}(\cdot)>0$ for $\lambda>\lambda^{z}(v)$. Notice also that, for $\lambda \in\left(\lambda^{z}(v), \beta\right), \gamma_{\lambda \lambda}<0$. Since $h(\cdot)$ and $\frac{\partial a_{c}(\cdot)}{\partial \lambda}$ are continuous functions, we can have $v^{\prime}$ and $\lambda^{\prime}$ such that:

$$
\lambda^{\prime}=\gamma\left(\lambda^{\prime}, v^{\prime}, 1\right) \text { and } \gamma_{\lambda}\left(\lambda^{\prime}, v^{\prime}, 1\right)=1
$$

Assuming that $a_{c}\left(1, v_{2}, 1\right)>\alpha$ (we can pick $\alpha$ close enough to zero), we can define two functions: $\lambda_{m}:\left[v^{\prime}, v_{2}\right] \rightarrow\left[0, \lambda^{\prime}\right]$ and $\lambda_{M}:\left[v^{\prime}, v_{2}\right] \rightarrow\left[\lambda^{\prime}, \lambda^{\prime \prime}\right]$, such that:

$$
\lambda_{j}(v)=\gamma\left(\lambda_{j}(v), v, 1\right) \text { for } j=m, M
$$

Claim: The functions in equation (43) exist and are well defined.

Proof. Note that for any $v \in\left(v^{\prime}, v_{2}\right): \lambda^{z}(v)>\gamma\left(\lambda^{z}(v), v, 1\right) ; \gamma\left(\lambda^{\prime}, v, 1\right)>$ $\lambda^{\prime}$ and $\gamma(1, v, 1)<1$. Then, due to the continuity of $\gamma$, for each $v$, there exist two functions $\lambda_{m}$ and $\lambda_{M}$, such that they are the fixed points of equation (43). Moreover, $\lambda_{m}(v) \in\left(\lambda^{z}(v), \lambda^{\prime}\right)$, and $\lambda_{M} \in\left(\lambda^{\prime}, 1\right)$. tion.

Claim: $\lambda_{m}(v)$ is a decreasing function and $\lambda_{M}(v)$ is an increasing func- 
Proof. For $\lambda_{m}(v)$, suppose not. Take $w_{1}<w_{2}$. Then, for any $\lambda \in$ $\left(\lambda_{m}(v), \lambda^{\prime}\right), \gamma\left(\lambda, w_{1}, 1\right)>\lambda$. But, $\gamma\left(\lambda_{m}\left(w_{2}\right), w_{2}, 1\right)>\gamma\left(\lambda_{m}\left(w_{2}\right), w_{1}, 1\right)>$ $\lambda_{m}\left(w_{2}\right)$. A contradiction.

For $\lambda_{M}(v)$, suppose not. Take $w_{1}<w_{2}$. Then, for any $\lambda \in\left(\lambda^{\prime}, \lambda_{M}(v)\right)$, $\gamma\left(\lambda, w_{1}, 1\right)>\lambda$. But, $\gamma\left(\lambda_{M}\left(w_{2}\right), w_{2}, 1\right)>\gamma\left(\lambda_{M}\left(w_{2}\right), w_{1}, 1\right)>\lambda_{M}\left(w_{2}\right)$. A contradiction.

Those fixed points will be equilibria if and only if $\lambda_{j}(v)<R(v)$, where we can define $R(v)$ as:

$$
R(v)=\frac{\delta v}{\phi}
$$

Assume that $v^{\prime}$ is such that $\lambda^{\prime}>\frac{\delta v^{\prime}}{\phi}$. Then, at $v^{\prime}$, the only equilibrium is given by $\lambda=0$. Since $\lambda_{m}(v)$ is decreasing, $R(v)$ is increasing and $R\left(v_{2}\right)>$ $\lambda_{m}\left(v_{2}\right)=0$. Then, there exists a level of wage $w^{\prime}$ such that $R\left(w^{\prime}\right)=\lambda_{m}\left(w^{\prime}\right)$. At $w^{\prime}$, there are two equilibria: $\lambda=0$ and $\lambda=R\left(w^{\prime}\right)=\lambda_{m}\left(w^{\prime}\right)$.

For any $v \in\left(w^{\prime}, v_{2}\right)$ there are three equilibria: $\lambda=0, \lambda=\lambda_{m}\left(w^{\prime}\right)$, and $\lambda=\min \left\{R(v), \lambda_{M}(v)\right\}$. The first two equilibria are obvious. We need to show that the third equilibrium exists. Then, we will show that for any $v \leq v_{2}, R(v)<\lambda_{M}(v)$. First, suppose that $R(v)<\lambda_{M}(v)$. Then, $\lambda_{M}(v)$ is not an equilibrium. Since $\gamma(R(v), v, 1)>R(v)$, there exists $r=r_{0}(R(v))$ such that $\gamma\left(R(v), v, r_{0}(R(v))\right)=R(v)$. The continuity of $\gamma(\cdot)$ guarantees the existence of such interest rate. See Section 4 for a proper definition of $r_{0}(R(v))$. Now, suppose that $\lambda_{M}(v)<R(v)$. Then, by definition, $\lambda_{M}(v)$ is an equilibrium. Since $\gamma(R(v), v, 1)<R(v)$, then $R(v)$ can not be an equilibrium. Note that, at $v=v_{2}, \lambda_{m}\left(v_{2}\right)=0$. That means that at $v=v_{2}$, there are only two equilibria.

It is easy to see that, in fact, $R(v)<\lambda_{M}(v)$ for all $v<v_{2}$. First, define $v^{\prime \prime}$ as: $a_{c}\left(1, v^{\prime \prime}, 1\right)=\alpha$. It is trivial to show that $v^{\prime \prime}>v_{2}$. Then, we can extend the domain of the function $\lambda_{M}(v)$ from $\left[v^{\prime}, v_{2}\right]$ to $\left[v^{\prime}, v^{\prime \prime}\right]$. Obviously, $\lambda_{M}\left(v^{\prime \prime}\right)=1$ and $R\left(v^{\prime \prime}\right)<1$. Now, since $\lambda_{M}(v)$ and $R(v)$ are both increasing and (weakly) concave functions, then:

$$
R(v)<\lambda_{M}(v) \text { for all } v \in\left(v^{\prime}, v^{\prime \prime}\right)
$$

(iii) First, note that for any $v>v_{2}, \gamma(0, v, 1)>0$. The bad equilibrium vanishes from the economy. Now, since we have proved in (ii) that $R(v)<\lambda_{M}(v)$, then the only equilibrium in this stage will be given by: 
$\lambda_{t}=\frac{\delta v_{t}}{\phi}$ and $r_{t, t+1}=r_{0}\left(\frac{\delta v_{t}}{\phi}\right)$. Note that when $v=\frac{\phi}{\delta}, \lambda_{t}=1$ and $r_{t, t+1}=1$, for all $t$.

Proof of Proposition 5: Let us denote by $\Pi_{g}$ and $\Pi_{b}$, the probabilities of choosing the good and the bad equilibrium, respectively, with $\Pi_{g}+\Pi_{b}<1$. Then, the variance of the equilibrium value, for a given $v \in\left(w^{\prime}, v_{2}\right)$, is given by the next equation:

$$
\begin{gathered}
V(\lambda(v))=\left(1-\Pi_{g}-\Pi_{b}\right)\left(\Pi_{g}+\Pi_{b}\right)\left(\lambda_{m}(v)\right)^{2}+\left(1-\Pi_{g}\right) \Pi_{g}(R(v))^{2} \\
-2\left(1-\Pi_{g}-\Pi_{b}\right) \Pi_{g} \lambda_{m} R(v)
\end{gathered}
$$

When $v$ is close to $w^{\prime}$, taking derivatives and assuming, for simplicity, that $\Pi_{g}=1-\Pi_{g}-\Pi_{b}$, we have that:

$$
\frac{\partial V(\lambda(v))}{\partial v} \cong 2 \Pi\left(1-\Pi-\Pi \frac{\delta w^{\prime}}{\phi}\right)\left(\frac{\delta}{\phi}+\frac{\partial \lambda_{m}(v)}{\partial v}\right)<0
$$

This is true since it can be showed that $\frac{\partial \lambda_{m}(v)}{\partial v} \rightarrow-\infty$ as $v \rightarrow w^{\prime}$. Now, if $v$ is close to $v_{2}$, and noting that $\frac{\partial \lambda_{m}(v)}{\partial v} \rightarrow 0$ as $v \rightarrow v_{2}$, we have that:

$$
\frac{\partial V(\lambda(v))}{\partial v} \cong 2 \Pi(1-\Pi) \frac{\delta}{\phi}>0
$$

Proof of Theorem 6: Assume, without loss of generality, that $v_{0}<w^{\prime}$. Then $\lambda_{1}=0$. Since $g_{l}(\delta)>w^{\prime}$, then with positive probability $\lambda_{2}=\frac{\delta v_{1}}{\phi}>$ $\lambda_{1}=0$. By induction, we can construct increasing sequences for $\lambda_{t}$ and $v_{t}$, with positive probability. Since $l_{s}(\lambda)$ is decreasing in $\lambda, g_{l}\left(l_{s}\right)$ is decreasing in $l_{s}$, and $g_{l}(\varepsilon) \rightarrow \infty$ as $\varepsilon \rightarrow 0$, at some point $\tau$, given $\lambda_{\tau}, v_{\tau} \geq \frac{\phi}{\delta}$. If such $\lambda_{\tau}$ exists with positive probability, then $\lambda_{\tau+1}=1$ with the same probability. Once the economy reaches this stage, for all $t \geq \tau+1, \lambda_{\tau}=1=\lambda^{*}$ and $v_{t}=f_{l}(\delta, 1)=v^{*}$. At this point, $r_{t, t+1}=1$.

The behavior of the interest rate is as follows. If $\lambda_{t+1}=R\left(v_{t}\right)$, then $r_{t, t+1}=r_{0}\left(R\left(v_{t}\right)\right)$. If $\lambda_{t+1}<R\left(v_{t}\right)$, then $r_{t, t+1}=1$. If $R\left(v_{t}\right)>1$, then $\lambda_{t+1}=1$ and $r_{t, t+1}=1$. 


\section{Proof of Proposition 7:}

(i) Just note that the equilibrium will be given by: $\lambda_{t+1}=\max \left\{\frac{\delta v_{t}}{\phi}\right\}$; $r_{t, t+1}=r_{l}\left(\frac{\delta v_{t}}{\phi}\right)$ if $\frac{\delta v_{t}}{\phi}<1$ and $r_{t, t+1}=1$ if $\frac{\delta v_{t}}{\phi} \geq 1$; and $v_{t+1}=f_{l}\left(l_{a}\left(\frac{\delta v_{t}}{\phi}\right), \frac{\delta v_{t}}{\phi}\right)$. All the variables depend on just $v_{t}$.

(ii) Assume, without loss of generality, that $v_{0}=0$. Then, since $v_{1}>v_{0}$, we have $\lambda_{2}>\lambda_{1}$. We can continue this reasoning by induction. If $v_{t-1}<$ $v_{t}<\frac{\delta}{\phi}$, then $\lambda_{t}<\lambda_{t+1}$. Since $l_{s}(\lambda)$ is decreasing in $\lambda, g_{l}\left(l_{s}(\lambda)\right)$ is decreasing in $l_{s}$, and $g_{l}(\varepsilon) \rightarrow \infty$ as $\varepsilon \rightarrow 0$, at some point $\tau v_{t} \geq \frac{\delta}{\phi}$. Then, $\lambda_{t+1}=1$ and $v_{t+1}=f_{l}(\delta, 1)$. Then, for any $t \geq \tau+1, \lambda_{t}=\lambda^{*}$ and $v_{t}=v^{*}$. Notice that $r_{t, t+1}=r_{l}\left(\frac{\delta v_{t}}{\phi}\right)$ is strictly increasing when $v_{t}<\frac{\delta}{\phi}$. To prove this, just note that $\frac{\partial \Pi_{a}(\lambda)}{\partial \lambda}>0$ and $\frac{\partial \Pi_{s}(\lambda)}{\partial \lambda}<0$. When $v_{t}>\frac{\delta}{\phi}, r_{t, t+1}=1$. This drastic change in the interest rate is associated to a change in the regime: from a constrained to an unconstrained economy.

\section{Proof of Proposition 8:}

(i) Since, by assumption, $f(l, 0)-\phi>g(l)$ for all $l$; all the agents with wealth greater than $\phi$ (i.e. with ability greater than $\frac{\phi}{v_{t}}$ ) will pay the entry cost at $t$ and become entrepreneurs at $t+1$. Then:

$$
\lambda_{t+1}=1-H\left(\frac{\phi}{v_{t}}\right)
$$

Given $\lambda_{t+1}$, then $v_{t+1}=v\left(\lambda_{t+1}\right)$, where $v(\lambda)$ is the function defined in Proposition 2 .

(ii) Note that, in equation (52), $\lambda_{t+1}$ is an increasing function of $v_{t}$. Also, $v(\lambda)$ is an increasing function of $\lambda$. Given the condition for growth in this economy, $v_{1}=g_{l}(\delta)>\frac{\phi}{\beta}$. Then, $\lambda_{2}=1-H\left(\frac{\phi}{g_{l}(\delta)}\right)>\lambda_{1}=0$. Following by induction, we can establish increasing sequences for $\lambda_{t}$ and $v_{t}$ when $v_{t}<\frac{\phi}{\alpha}$. Since $l_{s}(\lambda)$ is decreasing and $g_{l}(\varepsilon) \rightarrow \infty$ as $\varepsilon \rightarrow 0$, there exists some finite $\tau$ at which $v_{t} \geq \frac{\phi}{\alpha}$. From then on, $\lambda_{t}=1=\lambda^{*}$, for all $t$, and $v_{t}=f_{l}(\delta, 1)=v^{*}$, for all $t$. 
Figure I

\section{Per Capita GDP Variability for Group of Countries}

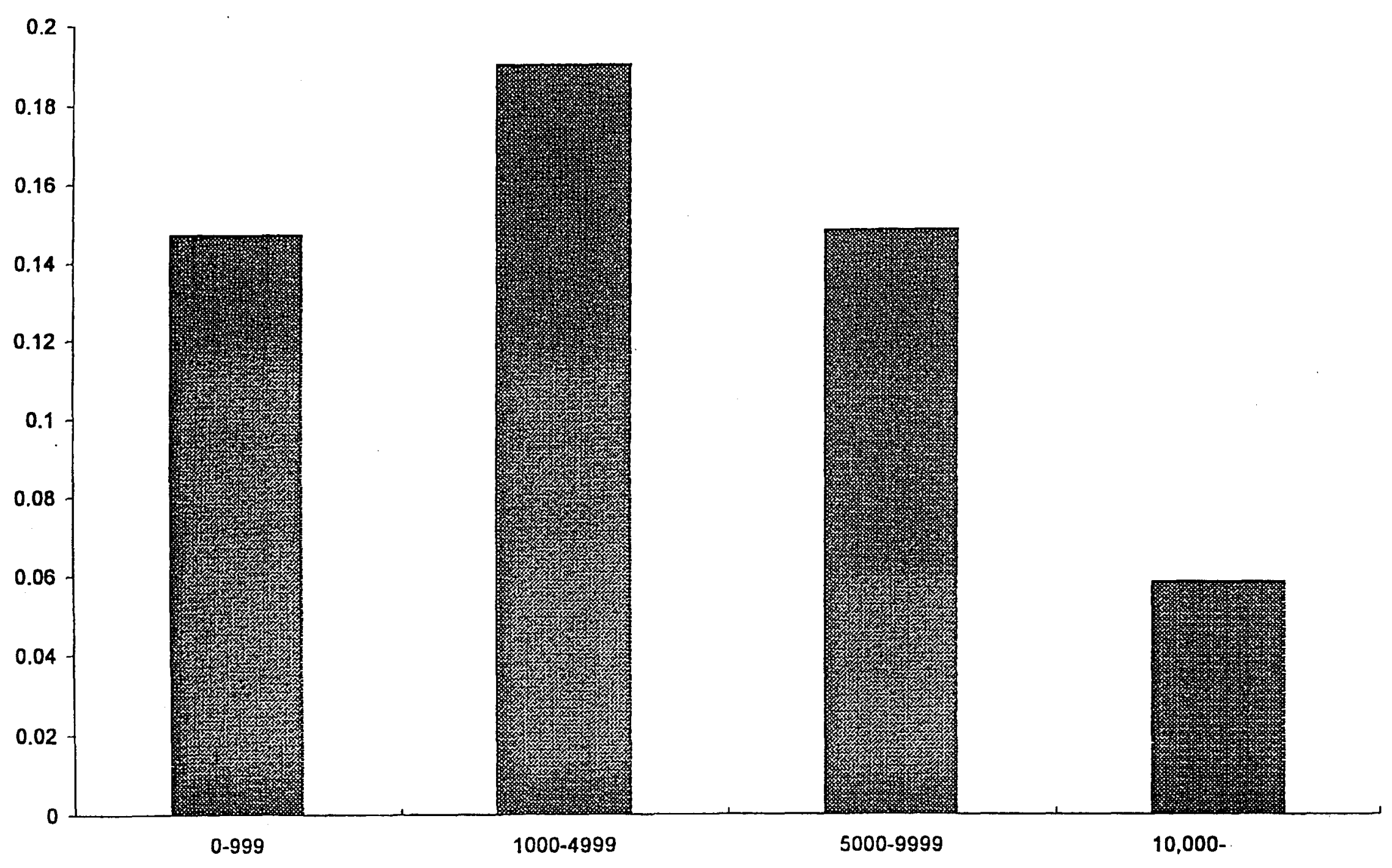

Source: Summers and Heston 
FIGURE 2

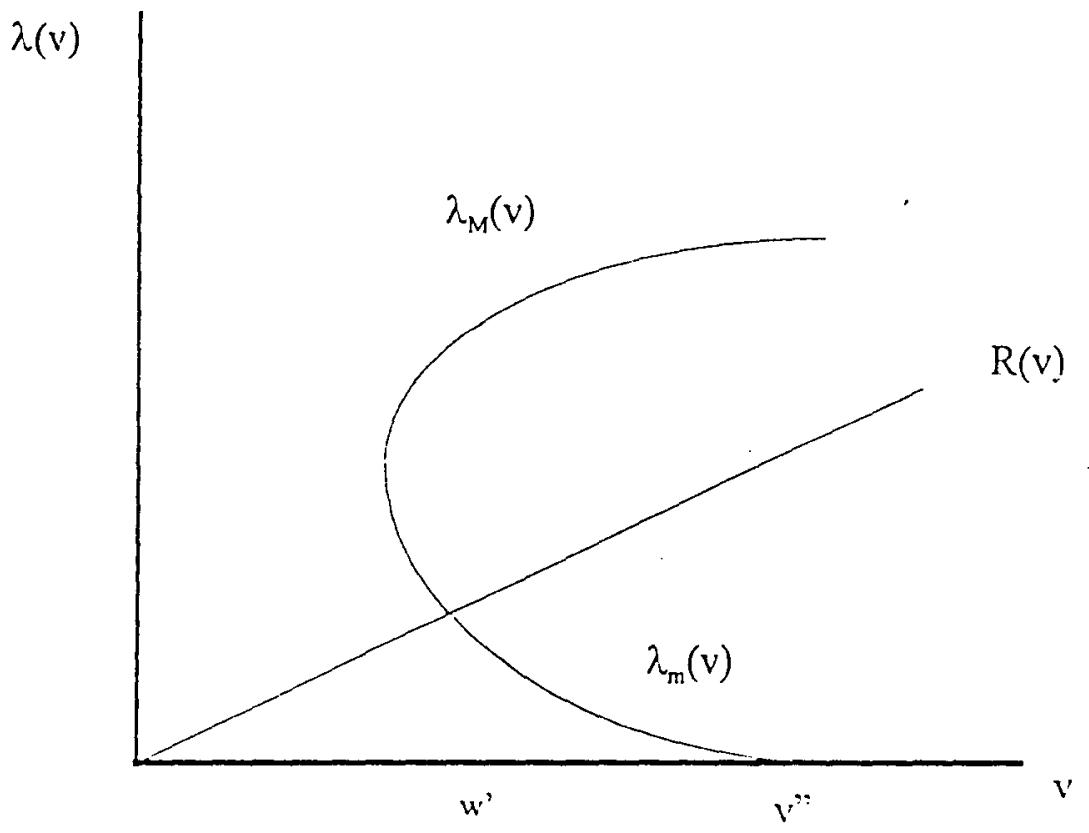




\section{WORKING PAPERS 1997}

\section{Business Economics Series}

97-18 (01) Margarita Samartín

"Optimal allocation of interest rate risk"

97-23 (02) Felipe Aparicio and Javier Estrada

"Empirical distributions of stock returns: european securities markets, 1990-95"

97-24 (03) Javier Estrada

"Random walks and the temporal dimension of risk"

97-29 (04) Margarita Samartín

"A model for financial intermediation and public intervention"

97-30 (05) Clara-Eugenia García

"Competing trough marketing adoption: a comparative study of insurance companies in Belgium and Spain"

97-31 (06) Juan-Pedro Gómez and Fernando Zapatero

"The role of institutional investors in international trading: an explanation of the home bias puzzle"

97-32 (07) Isabel Gutiérrez, Manuel Núnez Niekel and Luis R. Gómez-Mejía

"Executive transitions, firm performance, organizational survival and the nature of the principal-agent contract"

97-52 (08) Teresa García and Carlos Ocaña

"The role of banks in relaxing financial constraints: some evidence on the investment behavior of spanish firms"

97-59 (09) Rosa Rodríguez, Fernando Restoy and Ignacio Peña

"A general equilibrium approach to the stock returns and real activity relationship"

\section{Economics Series}

97-04 (01) Iñigo Herguera and Stefan Lutz

"Trade policy and leapfrogging"

97-05 (02) Talitha Feenstra and Noemi Padrón

"Dynamic efficiency of environmental policy: the case of intertemporal model of emissions trading"

97-06 (03) José Luis Moraga and Noemi Padrón

"Pollution linked to consumption: a study of policy instruments in an environmentally differentiated oligopoly"

97-07 (04) Roger Feldman, Carios Escribano and Laura Pellisé

"The role of government in competitive insurance markets with adverse selection" 